\title{
Genetic Etiologic Analysis in 74 Chinese Han Women with Idiopathic Premature Ovarian Insufficiency by Combined Molecular Genetic Testing
}

Jiandong Shen ${ }^{1}$, Dianyun $\mathrm{Qu}^{1}$, Yan Gao ${ }^{1}$, Fangxi Sun ${ }^{1}$, Jiazi Xie ${ }^{1}$, Xueping Sun $^{1}$, Dao Wang $^{2}$, Xiang $\mathrm{Ma}^{1}$, Yugui Cui ${ }^{1}$, Jiayin $\mathrm{Liu}^{3}$, and Feiyang Diao ${ }^{4}$

${ }^{1}$ Jiangsu Province Hospital and Nanjing Medical University First Affiliated Hospital

${ }^{2}$ The First Affliated Hospital of Nanjing Medical University

${ }^{3}$ The State Key Laboratory of Reproductive Medicine, Clinical Center of Reproductive Medicine, First Afliated Hospital, Nanjing Medical University

${ }^{4}$ First Affiliated Hospital with Nanjing Medical University

November 16, 2020

\begin{abstract}
Premature ovarian insufficiency (POI) refers to women premature amenorrhea before the age of 40, elevated levels of follicle stimulating hormone (FSH). In clinic, the etiology of POI remains unknown in most patients. In this study, seventy-four Chinese Han women with idiopathic POI were collected to analyze the genetic etiology. Triplet repeat-primed polymerase chain reaction (TP-PCR) was performed to screen the FMR1 (CGG)n premutation, and then 60 POI related genes were sequenced by targeted next generation sequencing (NGS) in POI patients with normal FMR1. One patient (1/74) with FMR1 premutation was identified. Targeted NGS revealed that $15.07 \%(11 / 73)$ patients had pathogenic or likely pathogenic variants of Mendelian genes (FOXL2, EIF2B2, CYP17A1, CLPP, MCM9, GDF9, MSH5, ERCC6, POLG). Ten novel variants in six Mendelian genes were identified, such as CLPP c.355A>C (p.I119L) and c.688A >C (p.M230L), MCM9 c.1157C > T (p.T386M) and c.1291A > G (p.M431V), GDF9 c. 238C >T (p.Q80X), MSH5 c.604G >C (p.G202R) and c.2063T >C (p.I688T), ERCC6 c.C1769C > T (p.P590L), POLG c.2832G >C (p.E944D) and c.2821A >G (p.I941V). This study enriched the variant spectrum of POI related genes and suggested targeted NGS was an efficient and optional further clinical test for POI patients without FMR1 premutation.
\end{abstract}

\section{Hosted file}

Targeted NGS in POI_Manuscript---HM-2020-11-08.pdf available at https://authorea.com/ users/376097/articles/493185-genetic-etiologic-analysis-in-74-chinese-han-women-withidiopathic-premature-ovarian-insufficiency-by-combined-molecular-genetic-testing 

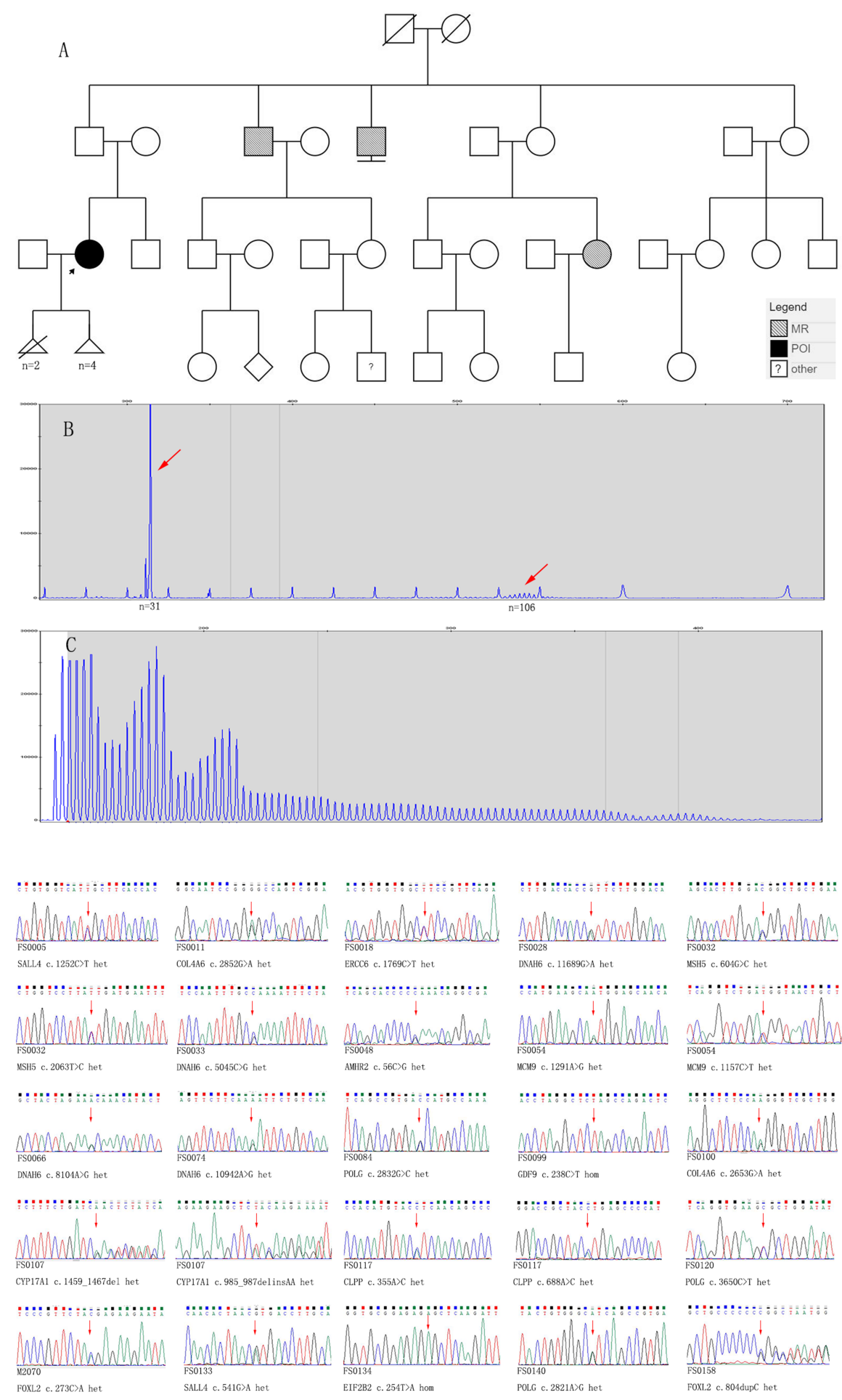

\section{Hosted file}

Table combined 1-3.pdf available at https://authorea.com/users/376097/articles/493185genetic-etiologic-analysis-in-74-chinese-han-women-with-idiopathic-premature-ovarianinsufficiency-by-combined-molecular-genetic-testing 